\title{
BMJ Open Multimorbidity of chronic non- communicable diseases and its models of care in low- and middle-income countries: a scoping review protocol
}

\author{
Fantu Abebe Eyowas (D) , ${ }^{1}$ Marguerite Schneider, ${ }^{2}$ Biksegn Asrat Yirdaw, ${ }^{2}$ \\ Fentie Ambaw Getahun ${ }^{1,3}$
}

To cite: Eyowas FA, Schneider M, Yirdaw BA, et al. Multimorbidity of chronic non-communicable diseases and its models of care in low- and middleincome countries: a scoping review protocol. BMJ Open 2019;9:e033320. doi:10.1136/ bmjopen-2019-033320

- Prepublication history and additional material for this paper are available online. To view these files, please visit the journal online (http://dx.doi org/10.1136/bmjopen-2019033320).

Received 30 July 2019 Revised 13 September 2019 Accepted 18 September 2019

Check for updates

(c) Author(s) (or their employer(s)) 2019. Re-use permitted under CC BY-NC. No commercial re-use. See rights and permissions. Published by BMJ.

${ }^{1}$ Department of Public Health, School of Public Health, College of Medicine and Health Sciences, Bahir Dar University, Bahir Dar, Ethiopia

${ }^{2}$ Alan J Flisher Centre for Public Mental Health, Department of Psychiatry and Mental Health, , University of Cape Town, Cape Town, South Africa

${ }^{3}$ Department of Public Health, School of Medicine, Addis Ababa University College of Health Sciences, Addis Ababa, Ethiopia

Correspondence to Mr Fantu Abebe Eyowas; fantuabebe@gmail.com

\section{ABSTRACT}

Introduction Multimorbidity is the coexistence of two or more chronic non-communicable diseases (NCDs) in a given individual. Multimorbidity is increasing in low- and middle-income countries (LMICS) and challenging health systems. Individuals with multimorbidity are facing the risk of premature mortality, lower quality of life and greater use of healthcare services. However, despite the huge challenge multimorbidity brings in LMICs, gaps remain in mapping and synthesising the available knowledge on the issue. The focus of this scoping review will be to synthesise the extent, range and nature of studies on the epidemiology and models of multimorbidity care in LMICs. Methods PubMed (MEDLINE) will be the main database to be searched. For articles that are not indexed in the PubMed, Scopus, PsycINF0 and Cochrane databases will be searched. Grey literature databases will also be explored. There will be no restrictions on study setting or year of publication. Articles will be searched using key terms, including comorbidity, co-morbidity, multimorbidity, multiple chronic conditions and model of care. Relevant articles will be screened by two independent reviewers and data will be charted accordingly. The result of this scoping review will be presented using the Preferred Reporting Items for Systematic Review and Meta-Analyses Extension for Scoping Reviews (PRISMA-ScR) checklist and reporting guideline.

Ethics and dissemination This scoping review does not require ethical approval. Findings will be published in peerreviewed journal and presented at scientific conferences.

\section{INTRODUCTION}

Multimorbidity is a relatively new concept in medical practice and research globally, ${ }^{1}$ and its definition and measurement are not universally established. ${ }^{2}$ Debates remain on the type of underlying diseases and the number of conditions to be included for the definition of multimorbidity. ${ }^{34}$

The most common definition of multimorbidity is 'the co-occurrence of two or more of chronic non-communicable diseases (NCDs) in a given individual'. ${ }^{45}$ Chronic conditions refer to a health problem requiring ongoing

\section{Strength and limitation of this study}

- To our knowledge, this is the first scoping review to map and synthesise multimorbidity studies and the available models of multimorbidity care in low- and middle-income countries (LMICs).

- This study will apply established scoping review methods.

- This review is limited to articles written in English only.

- However, it will attempt to provide a clear account of multimorbidity in LMICs without any restriction to publication year and status.

management over a period of years or decades (eg, hypertension, asthma, cancer, depression, diabetes and heart disease). The definition of multimorbidity, unlike comorbidity, does not account for the condition (index disease) that occurred at first in an individual. $^{6}$

\section{Epidemiology of multimorbidity in the global and LMICs context}

Global estimates of the prevalence of multimorbidity among different age groups vary from $14 \%$ to $90 \%$, with the highest prevalence in developed countries. ${ }^{78}$ This large variation in magnitude is unlikely to only be due to a real difference between populations but may also be due to methodological variability. ${ }^{9}$

In low- and middle-income countries (LMICs) on the contrary, the magnitude of multimorbidity was estimated to be between $14 \%$ and $68 \%^{10-13}$ and is expected to rise over the coming years. ${ }^{1}$

\section{Risk factors of multimorbidity}

Demographic changes, such as population ageing and advances in medical research and healthcare globally, are leading people to live longer and develop long-term 
disease conditions, including multimorbidity of chronic illnesses. ${ }^{114}$

The risk and prevalence of multimorbidity have consistently been associated with age. ${ }^{15}$ The continuing increases in life expectancy in high-income countries (HICs) and the large reductions in mortality at younger ages, particularly during childhood and childbirth, and from infectious diseases in LMICs led people living into their $60 \mathrm{~s}$ and beyond. With increasing age, numerous underlying physiological changes occur and the risk of chronic disease rises with an increased risk of experiencing more than one chronic condition at the same time. $^{16}$

Many studies have also shown that multimorbidity is socially patterned: people living in contexts of higher socioeconomic deprivation develop multimorbidity much earlier in life-up to 15 years earlier-compared with their wealthier counterparts. ${ }^{1718}$

Individual lifestyle factors, including obesity, inactivity, smoking and inappropriate use of alcohol; psychosocial factors, such as negative life events, external locus of control and small social network; mental health problems such as depression and long-term treatments, such as being on antiretroviral therapy, are also factors leading to multimorbidity. ${ }^{19-21}$

Although less consistent, there is growing evidence on the gender differences in the prevalence of multimorbidity. ${ }^{21}$ Most studies have shown that women are more likely than men to have higher risks of multimorbidity. ${ }^{223}$

\section{Impact of multimorbidity on individuals, family and the health system}

NCD multimorbidity impacts at individual, family and health system levels. ${ }^{24}$ Its implication for individuals is profound, including death at younger age,${ }^{25-27}$ impairments of physical and social functioning, ${ }^{2328}$ poor quality of life ${ }^{29}$ mental health problems, ${ }^{23} 30$ facing high cost of care $^{31}$ and higher rates of adverse effects of treatment and complex interventions. ${ }^{32}$

The impact at the health system level is recognised globally. ${ }^{2433}$ The management of multimorbidity is much more complicated and demanding for the health system, patients and their family compared with those patients having a single NCD. People living with multimorbidity need more holistic, generalist long-term care and support than patients having a single $\mathrm{NCD}^{34}$ and are high utilisers of healthcare resources due to difficult-to-manage conditions. ${ }^{35}$ However, the current model of care and guidelines, developed at a time when single disease frameworks were predominant, tends to focus on diseases in isolation rather than the needs and circumstances of the person with complexity care needs. ${ }^{21}$

Moreover, the rising tide of multimorbidity in LMIC is posing significant challenges to the health system, which is poorly configured and already burdened with communicable diseases (including epidemics), and maternal, neonatal and nutritional health problems. ${ }^{1}$ However, a synthesised evidence on the epidemiology of multimorbidity in LMICs is not available to the best of our knowledge.

\section{Models of multimorbidity care}

The United Nations' Sustainable Development Goal 3 requires countries to move towards ensuring that all people and communities have access to health services that are of high quality, integrated and people-centred. ${ }^{3637}$

Approaches to multimorbidity care are being generated and tested in HICs. However, it has been impossible to generate an ideal model of care for every possible combination of chronic conditions, ${ }^{34} 38$ and there is no consensus on the ideal approach that could satisfy the needs of patients across contexts. ${ }^{39} 40$ Some of the overarching principles in common across these different approaches are the notions of integration and coordination of care, patient-centred interventions and optimisation of medication therapy. ${ }^{24} 3441$

Models such as the patient-centred medical homes, ${ }^{42}$ the Salford Integrated Care Programme, ${ }^{43}$ the whole system intervention (CARE Plus) ${ }^{44}$ and patient activation system ${ }^{45}$ were reported to be effective in improving outcomes of patients with multimorbidity in HICs. However, other approaches such as the dimension of care, depression and drugs (3D) model ${ }^{46}{ }^{47}$ the telemonitoring in community centres mode ${ }^{48}$ and the patient-centred care model ${ }^{49}$ did not show a significant benefit to improve either quality of life or disease outcomes for patients with multimorbidity.

Nevertheless, a sensitive model of multimorbidity care appropriate for the context of LMICs has not yet been established. Applying the best available model from HICs to an LMICs is not feasible as primary care is organised in different ways across countries and even within different regions of one country. ${ }^{50}{ }^{51} \mathrm{~A}$ recent synthesis of chronic care models in LMICs did not address issues of multimorbidity care of NCDs. ${ }^{52}$

The information gap in terms of the epidemiology of multimorbidity in LMICs and extent to which the existing models of care are meeting the needs and expectation of patients with multimorbidity is huge. In addition, there is no synthesised evidence on whether any of the new models of multimorbidity care approaches or guiding principles have been implemented and assessed for their effectiveness in the LMICs setting. ${ }^{13}$

A precise review of evidence on the epidemiology of multimorbidity and the nature, extent of implementation and effectiveness of model of multimorbidity care in LMICs is instrumental to shape the focus of future research endeavours.

Scoping reviews are important for scanning the body of literature on a given topic and summarising and disseminating research findings, identifying research gaps and making recommendations for future research. ${ }^{53}$

The aim of this scoping review is to advance the state of knowledge of epidemiology of multimorbidity and models of multimorbidity care in LMICs. This synthesis 
will help to identify research gaps and shape future areas of investigation.

\section{Objectives}

The objectives of this scoping review are to:

- Map the available evidence on the prevalence and risk factors of multimorbidity in LMICs

- Review the existing models of multimorbidity care in LMICs.

- Identify gaps in evidence of the epidemiology of multimorbidity and its models of care in LMICs.

\section{METHODS AND ANALYSIS}

\section{Protocol design}

Methods for this study are developed based on Arksey and O'Malley's scoping review methodology ${ }^{54}$ and Levac et $a l$ 's and Peters $e t a l$ s scoping methodology enhancement recommendations. ${ }^{53} 55$ Accordingly, the following steps will be followed while undertaking this scoping review:(1) identifying the research question;(2) identifying relevant studies; (3) selecting studies; (4) charting the data; (5) collating, summarising and reporting the results and (6) consulting with relevant stakeholders.

\section{Framework stage one: identifying the research question}

Through consultation with the research team, the main research question is crafted as: What is known about the epidemiology of multimorbidity and its model of care in LMICs? This question would allow us to broadly review and capture the full breadth of existing literature, while providing opportunity for guiding research questions to be added or modified through an iterative process.

We identified the following secondary questions to guide the subsequent stages of the scoping review and to complement the broader research question above. These questions will include (1) What types of epidemiological research on multimorbidity have been conducted in LMICs? (2) In which LMICs were multimorbidity studies conducted? (3) Which target populations have been involved in previous multimorbidity studies? (4) What were the study setting (facility based or community based)? (5) What chronic physical and mental conditions have been identified? (6) What is the prevalence of multimorbidity in LMICs? (7) How was multimorbidity defined and measured? (8) What kinds of risk factors have been identified? (9) What are the existing models of multimorbidity care? (10) What were the proposed and effective models of multimorbidity care in LMICs? and (11) What were the limitations of the multimorbidity studies conducted in LMICs?

\section{Framework stage two: identifying relevant studies}

\section{Search strategy and information sources}

PubMed (MEDLINE) will be the main database to be searched. For articles that are not indexed in the PubMed, Scopus, PsycINFO and Cochrane databases will be searched. For grey literature, Google scholar, WHO's clinical trial register, WorldCat, Open Grey, Global Index
Medicus and LILACS will be explored. Additionally, we will hand search the reference lists of all literature deemed relevant. Search results will be downloaded into a citation manager and imported to an EndNote library for further review and to identify duplicates.

The search terms to be used for epidemiology of NCDs multimorbidity include comorbidity, co-morbidity, multimorbidity, chronic conditions, chronic disease, noncommunicable diseases, non-communicable diseases, adults and low- and middle-income countries.

The search terms used for the models of multimorbidity care include design of care, patient care management, patient management, primary healthcare, model of care, patient care, progressive patient care, patient care team, patient care planning, continuity of patient care and comorbidity or co-morbidity or multimorbidity or multiple chronic conditions or multiple chronic disease or noncommunicable diseases or non-communicable diseases, adults and low- and middle-income countries. We used different synonyms of LMICs and the World Bank Country and Lending Groups 2018 fiscal year list of LMICs (https://blogs.worldbank.org/opendata/newcountry-classifications-income-level-2017-2018). A draft of the full search strategy and data charting form are submitted as online supplementary file along with this manuscript.

Literature search will be conducted by experienced research team members. FAG is an expert and trainer of literature search and systematic reviews. BAY has completed a 5-day intensive training on literature searches and systematic reviews. Moreover, we will be using established methods to ensure quality of the literature search, screening and information charting.

\section{Framework stage three: study selection}

We will be employing two levels of screening: (1) a title and abstract review and (2) full-text review. Two investigators will independently screen the title and abstract of all retrieved citations for inclusion against a set of minimum inclusion criteria identified. Articles deemed relevant by either or both of the reviewers will be included in the fulltext review. In the second step, the two investigators will then independently assess the full-text articles to determine if they meet the inclusion/exclusion criteria. Any discordant full-text articles will be reviewed a second time and further disagreements about study eligibility at the full-text review stage will be resolved through discussion. A third reviewer may be consulted for arbitration of any discordant decisions at this phase. Reviewers will meet at the beginning, midpoint and final stages of the abstract review process to discuss any challenges or uncertainties related to study selection and to go back and refine the search strategy as needed. Throughout the review process, the number of studies included or excluded will be recorded. To establish agreement among the team, calibration exercise will be conducted on 25 randomly selected articles before screening and charting. A statistical measure will be employed to assess inter-rater 
reliability and the internal validity of our study's selection strategy. A minimum of $80 \%$ agreement between coders has been considered an acceptable threshold. ${ }^{56}$ The review process will be documented in sufficient detail to enable the study to be replicated by others.

\section{Inclusion criteria for studies on the epidemiology of multimorbidity}

We will be employing broad inclusion criteria to identify studies that measure the magnitude of multimorbidity, patterns of disease clusters, associated risk factors and impact of multimorbidity on patients in LMICs.

The following inclusion criteria must be met: (1) studies must refer to the multimorbidity of chronic illness in any clinical or non-clinical settings among adult population ( $\geq 18$ years old); (2) studies must investigate, assess or report the epidemiology of multimorbidity; (3) studies must be published in English.

With regard to study designs for the epidemiology of multimorbidity review, we will include prospective and retrospective cohort studies, case-control or nested case-control studies and cross-sectional studies. In addition, reference list of systematic reviews and scoping studies will be referred to search for further studies.

Inclusion criteria for the models multimorbidity care: (a) Studies must have assessed, implemented or reported effectiveness and outcome of multimorbidity care or any existing model of multimorbidity care in LMICs; (b) Studies that recommended guiding principles or approaches for designing effective models of multimorbidity care in LMICs.

Studies will be included if they describe concepts/principles or models of multimorbidity care (eg, coordination of care, integration of care, patient-centred care, holistic care, generalist care, collaborative care and quality of multimorbidity care), outcome of multimorbidity care or impact of multimorbidity on health system in LMICs.

With regard to study designs for the model of multimorbidity care, we will include randomised controlled trials, quasi-experimental studies, prospective and retrospective cohort studies, case-control or nested case-control studies and cross-sectional studies. Reference lists of systematic reviews and scoping studies will be referred to look for studies that are not identified in the primary search.

\section{Exclusion criteria for both the epidemiology and models multimorbidity care}

Studies from HICs, study protocols, commentaries, editorials and case reports will be excluded from this scoping review.

Although the mentioned inclusion and exclusion criteria will remain strict, we may iteratively define additional criteria as the research team become increasingly familiar with the subject matter through the full review of studies.

As quality assessment is not a typical requirement of scoping reviews, ${ }^{57} 58$ we will not apply a quality assessment of the articles to be reviewed. However, to enhance accuracy and completeness of the evidence-based search, we will be employing the elements of the Peer Review of Electronic Search Strategies (PRESS EBC Elements). ${ }^{59}$

\section{Patient and public involvement}

No patient will be involved.

\section{Framework stage four: charting the data}

The data collection instrument is developed both for the magnitude and model of multimorbidity care by the study team to extract data from the included studies. The team collectively developed the data charting form and determined the variables and extent of detail of the information to be extracted. To ensure that the instrument is capturing the information accurately, the study team will pilot the tool before the beginning of the review. For the epidemiology review, the data abstracted will include demographic information, methodology, setting, epidemiological findings and study limitations. For the model of care, the data abstracted will include demographic information, methodology, setting, intervention details, all reported patient-important outcomes and study limitations. Data abstraction will be conducted by two independent reviewers and abstracted data will be compared. Any discrepancies will be discussed to ensure consistency between the reviewers. Based on the recommendation by Arksey and O'Malley ${ }^{54}$ and Peters et al's guidance for scoping review, ${ }^{53}$ the data mining process will involve descriptive analytical methods that summarise and synthesise information in a transparent way.

A draft charting form is developed based on the Joanna Briggs Institute (JBI) scoping review manual ${ }^{60}$ and Peter et als scoping review guidance ${ }^{53}$ as part of this protocol to record characteristics of the included studies and the key information relevant to the review question. Information on the extraction field will include author(s), year of publication, source origin/country of origin, aims/purpose, study population and sample size, methodology, intervention type/model of care (if available), comparator, duration of the intervention (if applicable), outcomes if any, key findings that relate to the review question and limitations of the studies. We will be using EndNote to manage and code references in the conduct and reporting of this scoping review. ${ }^{61}$

\section{Framework stage five: collating, summarising and reporting} the results

As per the recommendation by Levac et $a l,^{55}$ we will describe stage five in three distinct steps: analysing the data, reporting results and applying meaning to the results.

The data will be summarised and reported in such a way that maps the breadth of existing literature within the field of multimorbidity and its model of care in LMICs. We will be mapping the concepts underpinning multimorbidity research and the type and aggregate quality of evidence available in the LMIC context. In addition, the available evidence on multimorbidity and its models 
of care in the context of LMICs will be mapped and described in detail. In order to facilitate usability of this scoping review, we will narrate the implications of findings within the broader context for research, policy and practice. Finally, we will provide an overview of the research field and where it currently stands and identify the gaps in knowledge that currently exist.

The output of our review will be presented using the Preferred Reporting Items for Systematic Review and Meta-Analyses Extension for Scoping Reviews (PRISMAScR) checklist and explanation format. ${ }^{62}$

\section{Study timeline}

The review team started preliminary searches in July 2019 and planned to complete the literature searching, screening and data charting over 2 months. Collating, summarising and reporting the results will then follow.

\section{Framework stage six: consulting with relevant stakeholders} Arksey and O'Malley ${ }^{54}$ indicated that consultation adds methodological rigour. Seeking stakeholders' (policy makers, practitioners and researchers in Ethiopia) perspectives and their views in applying the result to the scoping study will be considered once the preliminary findings from stage five are produced.

\section{DISSEMINATION AND ETHICS}

This study will constitute the first scoping review aimed at identifying the types of available evidence in multimorbidity of chronic diseases, clarifying key concepts in the literature and elucidating models of multimorbidity care and analysing knowledge gaps in LMICs. The result will be helpful for researchers to identify knowledge gaps in the field. The results from this scoping review will guide the next phase of development of multimorbidity studies in Ethiopia and LMICs at large. Since the scoping review methodology consists of reviewing and collecting data from publicly available materials, this study does not require ethics approval. ${ }^{63}$ We believe the proposed scoping review is feasible, attainable and timely. We will prepare presentations to disseminate findings to relevant stakeholder and end-user groups at local, national and international conferences. We will also publish our findings in a peer-reviewed journal.

We checked that there is no any registered review protocol on the same topic in International prospective register of systematic reviews (PROSPERO). As PROSPERO is not currently accepting scoping review protocols for registration, ${ }^{6062}$ this scoping review protocol was registered in JBI review database (http://joannabriggs.org/ research/registered_titles.aspx).

Acknowledgements We thank Bahir Dar University for the facilities we have used while facilitating this work.

Contributors The contributers statement should be revised as "FAE and FAG contributed to generating the concept of the review. FAE drafted the protocol. MS, FAG and BAY helped to edit the protocol. MS edited the language. All authors critically reviewed and approved the final manuscript for submission."
Funding The authors have not declared a specific grant for this research from any funding agency in the public, commercial or not-for-profit sectors.FAG and BAY receive support from AMARI (African Mental Health Research Initiative), which is funded through the DELTAS Africa Initiative (DEL-15-01). The DELTAS Africa Initiative is an independent funding scheme of the African Academy of Sciences (AAS)'s Alliance for Accelerating Excellence in Science in Africa (AESA) and supported by the New Partnership for Africa's Development Planning and Coordinating Agency (NEPAD Agency) with funding from the Wellcome Trust (DEL-15-01) and the UK government. The views expressed in this publication are those of the author(s) and not necessarily those of AAS, NEPAD Agency, Wellcome Trust or the UK government.

Competing interests None declared.

Patient consent for publication Not required.

Provenance and peer review Not commissioned; externally peer reviewed.

Open access This is an open access article distributed in accordance with the Creative Commons Attribution Non Commercial (CC BY-NC 4.0) license, which permits others to distribute, remix, adapt, build upon this work non-commercially, and license their derivative works on different terms, provided the original work is properly cited, appropriate credit is given, any changes made indicated, and the use is non-commercial. See: http://creativecommons.org/licenses/by-nc/4.0/.

ORCID iD

Fantu Abebe Eyowas http://orcid.org/0000-0002-4440-028X

\section{REFERENCES}

$1 \mathrm{Xu} \mathrm{X}$, Mishra GD, Jones M. Mapping the global research landscape and knowledge gaps on multimorbidity: a bibliometric study. J Glob Health 2017;7:010414.

2 Xu X, Mishra GD, Jones M. Evidence on multimorbidity from definition to intervention: an overview of systematic reviews. Ageing Res Rev 2017;37:53-68.

3 Bromley CM-LS. Beyond a boundary-conceptualising and measuring multiple health conditions in the Scottish population. University of Edinburgh, 2016.

4 Kastner M, Cardoso R, Lai Y, et al. Effectiveness of interventions for managing multiple high-burden chronic diseases in older adults: a systematic review and meta-analysis. CMAJ 2018;190:E1004-12.

5 Wang L, Palmer AJ, Cocker F, et al. Multimorbidity and health-related quality of life (HRQOL) in a nationally representative population sample: implications of count versus cluster method for defining multimorbidity on HRQoL. Health Qual Life Outcomes 2017;15:7.

6 Wilson MG, Lavis JN, Gauvin F-P. Designing integrated approaches to support people with multimorbidity: key messages from systematic reviews, health system leaders and citizens. Healthc Policy 2016;12:91-104.

7 Garin N, Koyanagi A, Chatterji S, et al. Global multimorbidity patterns: a cross-sectional, population-based, Multi-Country study. $J$ Gerontol A Biol Sci Med Sci 2016;71:205-14.

8 Oni T, McGrath N, BeLue R, et al. Chronic diseases and multimorbidity--a conceptual modification to the WHO ICCC model for countries in health transition. BMC Public Health 2014;14:575.

9 Fortin M, Stewart M, Poitras M-E, et al. A systematic review of prevalence studies on multimorbidity: toward a more uniform methodology. Ann Fam Med 2012;10:142-51.

10 Wang SB, D'Arcy C, Yu YQ, et al. Prevalence and patterns of multimorbidity in northeastern China: a cross-sectional study. Public Health 2015;129:1539-46.

11 Nunes BP, Batista SRR, Andrade FB, et al. Multimorbidity: the Brazilian longitudinal study of aging (ELSI-Brazil). Rev Saude Publica 2018;52Suppl 2.

12 Lalkhen H, Mash R. Multimorbidity in non-communicable diseases in South African primary healthcare. S Afr Med J 2015;105:134-8.

13 Nimako BA, Baiden F, Sackey SO, et al. Multimorbidity of chronic diseases among adult patients presenting to an inner-city clinic in Ghana. Global Health 2013;9:61.

14 WHO. Who global dialogue on partnerships for sustainable financing of noncommunicable disease (Ncd) prevention and control, 2018.

15 Willadsen T, Jarbøl D, Reventlow S, et al. Multimorbidity and mortality: a 15-year longitudinal registry-based nationwide Danish population study. J Comorb 2018;8:1-9.

16 Koroukian SM, Schiltz NK, Warner DF, et al. Multimorbidity: constellations of conditions across subgroups of midlife and older individuals, and related Medicare expenditures. Journal of Comorbidity 2017;7:33-43. 
17 Huntley AL, Johnson R, Purdy S, et al. Measures of multimorbidity and morbidity burden for use in primary care and community settings: a systematic review and guide. The Annals of Family Medicine 2012:10:134-41.

18 Mercer SW, Zhou Y, Humphris GM, et al. Multimorbidity and socioeconomic deprivation in primary care consultations. Ann Fam Med 2018;16:127-31.

19 Mounce LTA, Campbell JL, Henley WE, et al. Predicting incident multimorbidity. Ann Fam Med 2018;16:322-9.

20 Violan C, Foguet-Boreu Q, Flores-Mateo G, et al. Prevalence, determinants and patterns of multimorbidity in primary care: a systematic review of observational studies. PLoS One 2014;9:e102149.

21 Mercer S, Salisbury C, Fortin M. Abc of multimorbidity. First Edition. UK: John Wiley \& Sons, Ltd, 2014: 1-4.

22 Violán C, Roso-Llorach A, Foguet-Boreu Q, et al. Multimorbidity patterns with k-means nonhierarchical cluster analysis. BMC Fam Pract 2018;19:108.

23 Barnett K, Mercer SW, Norbury M, et al. Epidemiology of multimorbidity and implications for health care, research, and medical education: a cross-sectional study. The Lancet 2012;380:37-43.

24 Multimorbidity $\mathrm{AH}$. Understanding the challenge. In: A report for the Richmond group of charities, 2018.

25 Olaya B, Domènech-Abella J, Moneta MV, et al. All-Cause mortality and multimorbidity in older adults: the role of social support and loneliness. Exp Gerontol 2017;99:120-6.

26 Wei MY, Mukamal KJ, Multimorbidity MKJ. Multimorbidity, mortality, and long-term physical functioning in 3 prospective cohorts of community-dwelling adults. Am J Epidemiol 2018;187:103-12.

27 Martín-Lesende I, Recalde E, Viviane-Wunderling P, et al. Mortality in a cohort of complex patients with chronic illnesses and multimorbidity: a descriptive longitudinal study. BMC Palliat Care 2016;15:42.

28 Wijlhuizen GJ, Perenboom RJM, Garre FG, et al. Impact of multimorbidity on functioning: evaluating the ICF core set approach in an empirical study of people with rheumatic diseases. J Rehabil Med 2012;44:664-8.

29 Fortin M, Lapointe L, Hudon C, et al. Multimorbidity and quality of life in primary care: a systematic review. Health Qual Life Outcomes 2004;2:51.

30 Stubbs B, Koyanagi A, Veronese N, et al. Physical multimorbidity and psychosis: comprehensive cross sectional analysis including 242,952 people across 48 low- and middle-income countries. BMC Med 2016;14:189.

31 Picco L, Achilla E, Abdin E, et al. Economic burden of multimorbidity among older adults: impact on healthcare and societal costs. BMC Health Serv Res 2016;16:173.

32 Boyd CM, McNabney MK, Brandt N, et al. Guiding principles for the care of older adults with multimorbidity: an approach for clinicians: American geriatrics Society expert panel on the care of older adults with multimorbidity. J Am Geriatr Soc 2012;60:E1-25.

33 Moffat K, Mercer SW. Challenges of managing people with multimorbidity in today's healthcare systems. BMC Fam Pract 2015;16:129.

34 NICE. Multimorbidity: clinical assessment and management: Multimorbidity: assessment, prioritisation and management of care for people with commonly occurring multimorbidity. In: Nice guideline NG56: National Institute for health and care excellence, 2016.

35 Gauvin F-P, Wilson MG, Lavis JN, et al. Citizen brief: improving care and support for people with multiple chronic health conditions in Ontario. Hamilton, Canada: McMaster Health Forum, 2014.

$36 \mathrm{WHO}$. Framework on integrated. people-centred health services 2016.

37 WHO. Integrated care models: an overview; 2016.

38 Smith SM, Wallace E, O'Dowd T, et al. Interventions for improving outcomes in patients with multimorbidity in primary care and community settings. Cochrane Database Syst Rev 2016;9.

39 Bayliss EA, Bonds DE, Boyd CM, et al. Understanding the context of health for persons with multiple chronic conditions: moving from what is the matter to what matters. The Annals of Family Medicine 2014;12:260-9.

40 Smith SM, Soubhi H, Fortin M, et al. Managing patients with multimorbidity: systematic review of interventions in primary care and community settings. BMJ 2012;345.
41 Stokes J, Man M-S, Guthrie B, et al. The foundations framework for developing and reporting new models of care for multimorbidity. Ann Fam Med 2017;15:570-7.

42 Swietek KE, Domino ME, Beadles C, et al. Do medical homes improve quality of care for persons with multiple chronic conditions? Health Serv Res 2018;53:4667-81.

43 Bower P, Reeves D, Sutton M, et al. Improving care for older people with long-term conditions and social care needs in Salford: the classic mixed-methods study, including RCT. Health Serv Deliv Res 2018;6:1-188.

44 Mercer SW, Fitzpatrick B, Guthrie B, et al. The CARE Plus study - a whole-system intervention to improve quality of life of primary care patients with multimorbidity in areas of high socioeconomic deprivation: exploratory cluster randomised controlled trial and costutility analysis. BMC Med 2016;14:88.

45 Blakemore A, Hann M, Howells K, et al. Patient activation in older people with long-term conditions and multimorbidity: correlates and change in a cohort study in the United Kingdom. BMC Health Serv Res 2016;16:582.

46 Salisbury C, Man M-S, Bower P, et al. Management of multimorbidity using a patient-centred care model: a pragmatic cluster-randomised trial of the 3D approach. Lancet 2018;392:41-50.

47 Chaplin K, Bower P, Man M-S, et al. Understanding usual care for patients with multimorbidity: baseline data from a clusterrandomised trial of the $3 \mathrm{D}$ intervention in primary care. BMJ Open 2018;8:e019845.

48 Panagioti M, Reeves D, Meacock R, et al. Is telephone health coaching a useful population health strategy for supporting older people with multimorbidity? An evaluation of reach, effectiveness and cost-effectiveness using a 'trial within a cohort'. BMC Med 2018;16:80.

49 Spoorenberg SLW, Wynia K, Uittenbroek RJ, et al. Effects of a population-based, person-centred and integrated care service on health, wellbeing and self-management of community-living older adults: a randomised controlled trial on embrace. PLoS One 2018;13:e0190751

50 WHO. Multimorbidity: technical series on safer primary care; 2016.

51 Poitras M-E, Maltais M-E, Bestard-Denommé L, et al. What are the effective elements in patient-centered and multimorbidity care? A scoping review. BMC Health Serv Res 2018;18:446.

52 Lall D, Engel N, Devadasan N, et al. Models of care for chronic conditions in low/middle-income countries: a 'best fit' framework synthesis. BMJ Glob Health 2018;3:e001077.

53 Peters MDJ, Godfrey CM, Khalil H, et al. Guidance for conducting systematic scoping reviews. Int J Evid Based Healthc 2015;13:141-6.

54 Arksey H, O'Malley L. Scoping studies: towards a methodological framework. Int J Soc Res Methodol 2005;8:19-32.

55 Levac D, Colquhoun H, O'Brien KK. Scoping studies: advancing the methodology. Implementation Sci 2010;5.

56 Belur J, Tompson L, Thornton A, et al. Inter-Rater reliability in systematic review methodology: exploring variation in coder decision-making. Sociological Methods and Research 2018:1-29.

57 Munn Z, Peters MDJ, Stern C, et al. Systematic review or scoping review? guidance for authors when choosing between a systematic or scoping review approach. BMC Med Res Methodol 2018;18.

58 Pham MT, Rajić A, Greig JD, et al. A scoping review of scoping reviews: advancing the approach and enhancing the consistency. Res Synth Methods 2014;5:371-85.

59 CADTH. PRESS - Peer Review of Electronic Search Strategies: 2015 Guideline Explanation and Elaboration (PRESS E\&E), 2016.

60 Peters M, Godfrey C, Khalil H, et al. Scoping Reviews. Joanna Briggs Institute Reviewer's Manual. In: Joanna Briggs Institute reviewer's manual fourth ED. University of Adelaide: the Joanna Briggs Institute, 2017, 2017

61 Peters MDJ. Managing and coding references for systematic reviews and scoping reviews in endnote. Med Ref Serv $Q$ 2017;36:19-31.

62 Tricco AC, Lillie E, Zarin W, et al. PRISMA extension for scoping reviews (PRISMA-ScR): checklist and explanation. Ann Intern Med 2018;169:467-73.

63 Jolley RJ, Lorenzetti DL, Manalili K, et al. Protocol for a scoping review study to identify and classify patient-centred quality indicators. BMJ Open 2016;6. 